are of almost no value as indicating the efficacy or the disadvantages of any line of active treatment.

My experience of surgery in connexion with appendicitis is mil; overy case that has come under my care has hitherto made a complete recovery, with the solitary exception of a man admitted into the North West London Hospital some years ago suffering from a second attack. He was admitted with symptoms of general peritonitis and an operation was not considered advisable. $\mathrm{He}$ had apparently quite recovered from the primary attack. The treatment adopted before admission had been that of purging.

The onset of the affection varies much-it may be acutely sudden or creep insidiously on for several days. The following history is of value as it comes from the hand of a medical man who placed himself under my care in May, 1894. The patient stated: "On getting into bed I was suddenly seized with shivering and vomiting; my temperature was $1006^{\circ} \mathrm{F}$. The abdomen generally was tender. I passed a very restless night, nausea and retching being continuous. A bdowinal pain was acute, but so far not localised. The temperature was $101^{\circ}$. During the day symptoms were much the same. During the second night the pain became localised in the right iliac fossa and the diagnosis was clear." I saw the patient on the third day of illness. The right iliac fossa was occupied by a large tender tamour. The case did well, the bowels being relieved on the tenth day. There has been no return of the inflammatory mischief.

The symptoms may begin in a most indefinite manner and run a very slow course, in such cases being mostly gastric in character and liable to be treated as such.

[Dr. Hood here gave clinical descriptions of several other cases. He also quoted from Dr. Hawkins's monograph on Diseases of the Vermiform Appendix, and remarked that peritonitis occurring in connexion with the uterine appendages may sometimes create difficulties in the diagnosis. He continued :]

la the course of the last ifty years the treatment of these local inflammations occurring within the abdomen has passed through three stages. Daring the first period the main treatment was that of opium combined with calomel. Gradually the calomel was omitted from the prescription and treatment remained with opiam alone. During later years opium with many has been dreaded, and early operation has taken its place. For my own part I attach the utmost importance to the making of an early diagnosis and to the early use of opium; I scrupulously avoid all aperient remedies.

The cases which have been under my own care include twelve private patients and twenty-three in hospital, all of whom, with the one exception above alluded to, made a good recovery, and in no instance was there suppuration. The cases seen in private have remained under observation and have been free from any form of abdominal discomfort. Hospital cases do not afford evidence of the good results which follow a simple medical treatment, in consequence of the fact that a large proportion of them do not apply until long after the commencement of their symptoms. My patients, on the contrary, with scarcely an exception, came under treatment at a very early stage of illness, and are of value as showing the results of a purely medical treatment. Pactically a relapse is not of dangerous omen. Among my private cases in which the subsequent history has been known to me there were three instances of relapse. One was a child who sixteen years ago had two attacks within the same year and has had no return. The second was a man who suffered from a very severe form in 1879 and in $188 \mathrm{~L} \mathrm{had}$ another, but since that date has had no return or any form of sbdominal discomfort. The third was a joung man who during 1888-9 had five attacks, in 1890 passed through typhoid ferer and since the last attack of appendicitis has had no trouble of any kind referable to the abdomen. These cases were all treated with opium. The patient should be confined to bed and kept absolutely to slop diet - in fact, treated on the same lines as if suffering from typhoid fever. He should have hot applications to the abdomen, and from first to last should be rigorously kept from any form of aperient remedy. I have never seen any harm ensue from the bowels being confined for from seven to double that number of days. My plan has been to wait till the fall of temperature and then when scybalous masses are to be felt in the left iliac fossa to give a simple soap-and-water enema. Opium should be given from the first moment that diagnosis is possible. I prefer to administer it either in its solid form or as Dover's powder, the amount varying with the exigencies of the case, and I think that one is more likely to err from giving too little than from giving too much. In an ordinary case I give half a grain every four or six hours, and I have never seen harm ensue. It soothes the nerrous system, renders the restraint of bed more endurable, relieves the pain which if untreated is of ten very great, promotes physiological rest of the part affected, and, in my opinion, promotes repair and restoration of the inflamed tissue. When I first commenced practice I used to combine belladonna with the opium, but soon gave up the use of the former drug, as I could find no material advantage in the combination and it had the disadvantage of making the throat dry and parched.

\section{CASES OF SURGICAL TUBERCULOSIS TREATED BY KOCH'S NEW TUBERCULIN.}

BY FREDERIC EVE, F.R C.S. ENG.,

SURGEON TO THE LONDON BOSPITAL AND TO THE EVELINA HOSPITAL FOR SICK CHILDREN.

THE following eight cases are examples of surgical tuberculosis under my care at the Evelina Hospital in which the treatment by Koch's new tuberculin ${ }^{1}$ (tuberculin R) has been completed, or nearly so. The first three cases of tuberculous disease of joints without sinuses or evidence of softening of caseous material show the only favourable results. Cases 2 and 3 were examples of early tuberculosis of the elbow and hip respectively. Of these it may fairly be said that the same results might have been anticipated from "surgical rest" and improved diet \&c. In Case 1-a patient with relapsing tuberculosis of the knee of four years' duration-it seems probable that the remedy ezercised a favourable influence. Case 5 was an instance of tuberculous peritonitis with ascites which was treated immediately after admission by laparotomy. During the administration of the tuberculin there was no reaccumulation of the fluid, but the child continuously lost weight. It was necessary to give the tuberculin very slowly and gradually owing to the weakly condition of the child and to avoid pyrexia; so that at the date of writing the treatment is only just completed. The remaining four cases were all examples of taberculosis with sinuses. These, with one possible exception, were absolutely uninflaenced by treatment. The sinuses did not heal, and in three instances operation was ultimately required. Case 7, that of a child with tuberculous teno-synovitis of the dorsum of the hand, seems clearly to show that no immunity to tuberculosis was established by the treatment. The tuberculons material had become caseous and broken down with sinus formation, but was not, in the ordinary acceptance of the term, septic. After the conclusion of the trberculin course all the taberculous material was freely and carefally removed by operation, no bone disease being found. The wound healed by first intention, but two or three weeks later recrudescence occurred in the scar and subjacent tissues. The exception mentioned above was a patient with tuberculous epididymitis. A sinus remained after the opening of an abscess at the London Hospital just previously to admission to the Evelina Hospital. The sinus healed during the administration of tuberculin.

My own personal impressions ${ }^{2}$ of the results of the new remedy may be summed up as follows. Some slight al though no markedly favourable influence may be exerted in cases of early tuberculosis of joints or in those in which no evidence of softening of caseous material exists. But the effect where caseous material has broken down, and especially if the disease has become septic, is negative. The subsequent course of the cases described below will be carefully watched.

It cannot be said that the new remedy is altogether free from the drawbacks possessed by the old tuberculin of producing pyrexia and constitutional disturbance. In nearly

1 Professor Koch's original article announcing the remedy appeared in the Deutsche Medicinische Wochenschrift, April 1st, 1897.

2 My experience is not confined to the cases here recorded, but I have watched several other cases in which the treatment has not been completed and some under the care of my colleagues in which the course was completed. 
every case a considerable rise of temperature occurred after one or two milligramme doses had been reached, and sometimes before. After this the duplication of successive doses, recommended by Koch, had to be given up for a more gradual increase. The pyrexia in some cases was very great, amounting to a rise of $7^{\circ} \mathrm{F}$. Other unpleasant symptoms follow the administration of larger doses, such as vomiting, malaise, headache, and prostration. No local reaction was observed. In several instances abscesses formed at the seat of injection, although strict antiseptic precautions were enjoined. The number of these may in a measure be explained by the fact that during the progress of the trial we had several changes of resident officers. I am much indebted to our registrar, Dr. $\mathrm{O}$. $\mathrm{K}$. Williamson, for the following abstract of the notes of the cases; also to Miss Getz for the great help she afforded us in the administration of the remedy.

CASE 1. Thberculosis of the knee joint. - A girl, aged seven and a half years, was admitted to hospital on May 15th for a tuberculous knee. It was stated that the left knee became swollen and painful after a fall four years previously. Since then the swelling had increased. About a year before admission the knee became bent; it was straightened at the Dover Hospital and the limb was put in plaster-of-Paris. There were marked wasting of the left leg and swelling of the knee. The synovial membrane generaIly was much thickened and pulpy. Movements were extremely limited, but there was no displacement of the bones. On May 16th tuberculin ( $\frac{1}{5} \overline{0}$ milligramme) was injected. Sixteen doses in all were injected. The dose was doubled each time until 16 milligrammes was the amount reached; after this the dose administered was always 20 milligrammes. The injections were given at intervals of two days until $\frac{1}{2}$ milligramme was the amount reached. The intervals were then increased. Two doses of 20 milligrammes were injected. Sixty. four days was the period of time over which the injections were continued. As the result of the injections there was no local reaction. No marked rise of tempera. ture took place until the dose of tuberculin had reached 4 milligrammes. The temperature on the evening after injection rose to $102^{\circ} \mathrm{F}$. (a rise of nearly three degrees). The day after the injection of 8 milligrammes the temperature rose to $101 \cdot 2^{\circ}$ (two degrees rise). The day when 16 milligrammes were injected the temperature rose to $103.2^{\circ}$ (from normal). The day after the first injection of 20 milligrammes the temperature rose to $101.2^{\circ}$, and on the day of the second injection the temperature rose to $101^{\circ}$ (two degrees rise). After the injection of $16 \mathrm{milli}$ grammes the child was very sick once. After the first injection of 20 milligrammes the child romited several times, the face was pale, and she complained of headache; after the second injection of 20 milligrammes she was again sick and the face was very pale. On May 17th the knee was put $\mathrm{p} p$ in a Macintyre's splint, in which it remained until June 25th. On June 24 th the knee measured above the patella $8 \frac{7}{8}$ in. in circumference. On July 30 th the measurement was found to be $8 \frac{5}{8}$ in. There was no apparent thickening of the synovial membrane, except slight fulness at the lower part of the patella on each side. The patella was found to be fixed, there was no movement in the joint, and no pain on percussion or palpation. The child was sent to a convalescent home wearing a moulded leather splint.

Remarks.--In this case the disease was well established, being of four years' duration, but no softening had taken place. The improvement was undoubted, bat lapse of time must show if a permanent cure has been obtained. It is impossible to estimate in what measure respectively the improvement was due to rest, abundant diet, and nursing for seventy-six days, or to the tuberculin. Seeing that the disease was of such duration and that the joint had been treated at a seaside hospital I should be inclined to ascribe a large share of credit to the injections.

CAsw 2. Tuberculous disease of the elbow. - A girl, aged four years, was admitted on April 21st for tuberculous disease of the elbow joint (right). About September, 1896, the mother observed after the child had had a fall that she could not straighten her arm and that the elbow was swollen. There was very little movement in the joint; it could not be extended to more than $120^{\circ}$ or flexed beyond a right angle; rotation also was very much limited. The joint felt generally thickened. There was a distinct pulpy swelling in the neighbourhood of the radial articulation. An angular splint was applied. The child was healthy in appearanoe and well nourished. On May $8 \mathrm{th}{ }_{5}{ }^{\frac{1}{0}} \mathrm{~m}$ milligramme of tuberculin was injected. Eighteen doses in all were administered. The dose was doubled until 15 milligrammes were reached; four doses of 20 milligrammes were then successively given. The interval between successive doses was two days until 2 milligrammes were reached. There were then intervals of four days and three days until 8 milligrammes were reached. The intervals after this varied from eight to eleven days. Serenty-two days was the period of time over which the injections were given. As the result of the injections there was no marked rise of temperature until 15 milligrammes were injected. After this there was a rise on the same evening to $102^{\circ} \mathrm{F}$. (about four degrees). After the first injection of 20 milligrammes there was similarly a rise of temperature to $101 \cdot 2^{\circ}$ (from $97^{\circ}$ ). A rise occurred after the second injection of 20 milligrammes to $102^{\circ}$ from $974^{\circ}$; and after the third injection there was an almost exactly similar rise of temperature. The day after the last injection there was a rise to $1006^{\circ}$ from normal. After the second injection of 20 milligrammes an abscess was observed at the seat of injection on the left side of the abdomen. The patient had been fretful in the night and had vomited once. After the third injection she had profuse sweats over the head and face. These were the only effects observed. On May 27th the circumference of the elbow taken round the groove on the front of the elbow and over the point of the olecranon with the elbow at right angles was $6 \frac{7}{8}$ in. On Aug. 6 th the circumference was found to be $6 \frac{5}{8}$ in., and the pulpy swelling had disappeared. The loss of mobility remained practically the same, but there was neither the slightest pain on movement nor tenderness. The child's general condition on leaving the hospital appeared to be thoroughly sound; she was very fat. The arm was put up in plaster bandages. The progress of this case of early tuberculous disease was in every way satisfactory.

CASE 3. Hip joint aisease.-A boy, aged five years, was admitted with hip disease (left) on April 21st. The boy limped and complained of pain on walking. The limping was noticed a week after he fell from a seat in March last. There was some fulness in the left groin, and very little movement in the joint could be obtained. The boy was well nourished. On April 24th weight extension (4 1b.) was applied; on May 13th this was stopped and a double Thomas's splint was applied. On May 7th $\frac{1}{1000}$ milligramme was injected. Eighteen doses in all were injected. The second dose was $\frac{1}{2}$ milligramme; the dose was then doubled each time until 15 milligrammes were reached; after this four successive doses of 20 milligrammes were administered. Seventy-three days was the period of time over which the injections were continued. The interval between successive doses was usually two days until 2 milligrammes was the amount reached. The intervals after this were in order four, three, eight, eight, eight, eleven, and ten days. As the result of the injections there was no marked rise of temperature until $\frac{1}{4}$ milligramme was injected. On the evening after this there was a rise of temperature to $100.4^{\circ} \mathrm{F}$. (from $97^{\circ}$ ). The next marked rise of temperature was after the injection of 2 milligrammes. The day after this the temperature rose to $100^{\circ}$ (from normal). Again, the day after the injection of 4, milligrammes the temperature rose to $99.8^{\circ}$ (from' $97^{\circ}$ ). After the injection of 8 milligrammes there was a rise next morning to $1038^{\circ}$ (from $97^{\circ}$ ); and after the injection of 15 milligrammes there was a rise to $105 \cdot 6^{\circ}$ (from $97 \cdot 8^{\circ}$ ). After the first injection of 20 milligrammes there was a rise of temperature to $103.8^{\circ}$ (from $964^{\circ}$ ), after the second injection a rise from $97^{\circ}$ to $103^{\circ}$, after the third injection a rise from $97^{\circ}$ to $103.6^{\circ}$, and after the fourth injection a rise from normal to $103.2^{\circ}$. After the injection of 15 milligrammes he was sick several times, slept very little, the face and hands were occasionally cold, and he had profuse general sweats. After the first injection of 20 milligrammes he vomited a few times; after the second injection he vomited three times, his face was pale and he complained of pain in the abdomen after the third injection he had similar symptoms, and after the fourth injection he was once sick. On July 9 th there was still some fulness in the left groin. The joint admitted of a certain amount of movement without pain, especially flexion. The thigh could be flexed to an angle of $40^{\circ}$ with the horizontal. The boy left the hospital on a double Thomas's splint.

Remarks. - In this case it may be said that the same amount of improvement would have followed "rest" and general 
treatment in a large proportion of cases of disease of the hipjoint.

CASE 4. Caries of the tarsus.-A girl, aged six years, was admitted to hospital on Feb. 13th for a sinus discharging thin serous pus on the anterior surface of the left foot. Two and a half years ago the child went into the Forest Gate Infirmary with wasting. She was discharged in August, 1896, apparently well, with the exception of a discharging sore on the left foot. Since Christmas wasting and night sweats had been present. The sinus led to carious bone. The dorsum was swollen and pulpy. On March 12th the scaphoid, cuneiform bones, and cuboid were removed by two incisions on the outer and inner sides of the dorsum of the foot respectively. The bones of the foot were then brought together by means of deep catgut sutures, the wound was dressed with cyanide ganze, and the foot was placed in plaster splints. On admission there was also a large indurated swelling in the right lumbar region with a lateral curvature to the right. On April 6th, of the two granulating wounds on the dorsum and external surface of the foot respectively the outer one had healed and the inner had not. On the 28th a small abscess in the groin (right) was opened. At this date the wound in the foot was nearly healed, but one corner was rather inclined to break down. The child was thin and rather pale. On May $8 \mathrm{sh} \frac{1}{50}$ milligramme of tuberculin was injected. Eighteen doses in all were injected. The particulars with regard to the doses and the intervals between successive doses were precisely the same as in Case 6. As the result of the injections the temperature was not observed to rise until 1 milligramme had been injected. On the evening after this there was a rise from $97.4^{\circ}$ to $101^{\circ} \mathrm{F}$. On the day of injection of 8 milligrammes there was a rise from $97^{\circ}$ to $1014^{\circ}$. On the day of injection of 15 milligrammes there was a rise from normal to $104^{\circ}$. On the day of the first injection of 20 milligrammes there was a rise of temperature from normal to $103^{\circ}$; on the day of the second injection of 20 milligrammes there was a similar rise; on the day of the third injection of 20 milligrammes there was a rise of temperature from normal to $1012^{\circ}$; and on the day of the last injection there was a rise of temperature from normal to $1024^{\circ}$. After the injection of 15 milligrammes inflammation was observed at the seat of injection, she slept badly, complained of headache, and vomited several times. After the first injection of 20 milligrammes her face was pale and she romited once. An abscess formed at the seat of injection. After the second injection of 20 milligrammes she vomited several times; after the third and fourth injections there was, however, no sickness. On July 2 ad, after having had two injections of tuberculin of 20 milligrammes, the sinuses in the foot were scraped with a sharp spoon and some softened bone removed. The sinus in the right groin was opened and scraped. The edges of the wound were brought together with silkworm gut.

Remarks.-The local disease was apparently uninfluenced by the tuberculin. The child was sent to a convalescent home with a sinus still open on the dorsum of the foot.

$\mathrm{CASE}_{\mathrm{S}}$ 5. Tuberculous peritonitis (ascitic variety).-A female infant, aged seventeen months, was admitled on May 14th. The abdomen was greatly distended, with bulging in the flanks, and very tense; a fluid thrill was easily obtained. The child was plump and looked healthy; her colour was good. On June 3rd laparotomy was performed. Much serous fluid was let out from the peritoneal cavity and the peritoneum was found to be studded with tabercles. The operation wound healed well. On June 8 ih $\frac{1}{5} 0$ milligramme tuberculin was injected. The dose was very gradually increased, the fall 20 milligrammes not being reached until September. The child had lost $4 \mathrm{lb}$. $4 \mathrm{oz}$. since admission and looked pale. Distinct nodules were to be felt in the neighbourhood of the wound, but there was little if any reaccumulation of fluid. This patient was transferred to me for surgical treatment by my colleague, Dr. Willcocks.

CASE 6. Tuberculous dactylitis.-A girl, aged ten years, was admitted to hospital on April 24th for tuberculous dactylitis. There was thickening of the first phalanx of the ring finger of the left hand, the tissues over the thickened part were inflamed, and there was a large fungating ulcer on the posterior and ulnar surface of the finger. The patient's general condition was good. The forearm and hand were put up in an anterior splint. On May $8 \mathrm{th} \mathrm{h}_{\overline{\mathrm{o}} \mathrm{j}} \mathrm{m}$ milligramme of tuberculin was injected; eighteen doses in all were administered.
The dose was doubled each time of injection until 16 milligrammes was the amount injected. 'The next dose was 20 milligrammes, and the dose thenceforward was kept at this. The injections were given at intervals of two days until the dose of 2 milligrammes was reached; there was four days' interval between the injections of 2 and 4 milligrammes; three days between the injections of 4 and 8 milligrammes; seven days between the injections of 8 and 16 milligrammes; and nine days between the injections of 16 and 20 milligrammes. After this the intervals varied from eight to eleven days. The last dose was on July 19th. Seventy-two days was the period of time over which the injections were continued. Four doses of 20 milligrammes each were injected. The temperature did not rise until $\frac{1}{2}$ milligramme was injected. The same day it rose to $101^{\circ} \mathrm{F}$. (trom $984^{\circ}$ ). The day after 4 milligrammes were injected the temperature rose to $102 \cdot 2^{\circ}$, after 8 milligrammes to $102^{\circ}$, whilst the same day that 16 milligrammes were injected it rose to $104 \cdot 6^{\circ}$. On the day of the first injection of 20 milligrammes the temperature rose to $1038^{\circ}$, on the day of the second injection of 20 milligrammes to $105 \cdot 6^{\circ}$, on the night after the third injection to $103^{\circ}$, and on the day of the fourth injection to $1024^{\circ}$. After the injection of 8 milligrammes an abscess was observed at the seat of injection, with inflammation around it. After the injection of 16 milligrammes headache was observed coincidently with the rise of temperature. There were also redness and great tenderness at the seat of injection, and the patient was sick afterwards. After the first injection of 20 milligrammes the hands and face were observed to be cold coincidently with the rise of temperature. After the second injection she complained of headache, the face was very pale, and she was sick once, coincidently with a rise of temperature. On Jaly 30th an operation was performed. An incision was made over the dorsum of the first phalanx, and a sinus was found leading in to the interior of the shaft of the bone. Neither joint was affected. On the external surface of the ligaments of the first interphalangeal joint some recent tuberculous granulation tissue was found. The interior of the thickened shaft of the first phalanx was filled with diffluent caseous material. The operation wound healed per priman.

Remarks.-Soon after the commencement of the injections the ulcer partially healed and the swelling of the soft parts of the finger diminished. This may have been due to carefol antiseptic dressing, rest, and improved diet. Subsequently the condition of the finger became stationary.

CAsE 7. Tuberculous teno-synovitis. - A boy, aged tight years, was aämitted on June 1st for tuberculous disease of the dorsum of the hand, which had been affected on and off for three or four years, but had become worse lately. There was considerable thickening over the fourth metacarpal bone of the left hand, and some ulceration of the skin. The first phalanx of the ring finger was also considerably thickened, and there was a discharging sinus in the palm. The boy was delicate in appearance and decidedly thin. A palmar splint was applied. On Jane $4 t h \frac{1}{500}$ milligramme of tuberculin was injected. Fourteen doses in all were administered, the dose being doubled each time until 16 milligrammes were reached. The injections were made at intervals of two days until $\frac{1}{6} 0$ milligramme was reached. Then intervals of from three to eleven dass were allowed to intervene until 2 milligrammes were ixjected; then intervals of two days until 16 milligrammes was the amount reached. This was arrived at on Jaly 31st. Fifty-keven days was the period of time over which the injections were continued. As the result of the injection a rise of temperature to $103^{\circ} \mathrm{F}$. was observed the day after ${ }^{\frac{2}{2}}$ milligramme was injected (nearly three degrees rise). The day of injection of $\frac{7}{60}$ milligramme the temperature rose to $102^{\circ}$ (about three degrees). The next occurrence of marked rise of temperature was on the day after $\frac{1}{3}$ milligramme was injected, when there was a rise to $1022^{\circ}$ (about four degrees). The day after injection of $\frac{1}{2}$ milligramme there was a rise of temperature to $102.8^{\circ}$ (about five degrees), the day afier injection of 1 milligramme there was a rise of about fonr degrees, after 2 milligrammes to $102 \cdot 2^{\circ}$ (about three degrees) the next important rise being after 16 milligrammes to $104^{\circ}$ (from normal). No other effects were observed. On July 30 th the swelling on the dorsum of the hand was incised and found to contain diffluent caseous material connected with tendon sheaths but not with bone. A communication existed with the ulcer on the palmar surface.

Remarls. - Primary uri in took place; but from two to 
three weeks later the scar became tuberculous and broke down. As the result of treatment the swelling rather increased and the skin over the tuberculous granulation tissue broke down at several points. Two sinuses existed when the child came under observation, but there was practically no suppuration.

CASE 8. Tuberculous epididymitis.-A boy, aged ten years, was admitted to hospital with tuberculosis ot the epididymis on June 7th. There was a sinus on the left side of the scrotum, the skin around which was adherent to the left testicle. The sinus discharged serous flaid. Marked thickening of the epididymis existed, but the vas was normal. The boy, although thin, looked healthy. Previously to admission at the Evelina Hospital an abscess connected with the left testicle was incised at the London Hospital and the sinus remained. On June $8, \mathrm{~h}$. $\frac{1}{0}$ milligramme of tuberculin was injected. Nine doses in all were administered. The dose was doubled on each occasion the largest dose reached being $\frac{1}{2}$ milligramme. Fortythree days was the period of time over which the injections were continued. As the result of the injection the first marked rise of temperature was on the day after the injection of $1 \frac{1}{25}$ milligramme, when it rose from normal to $100.8^{\circ} \mathrm{F}$, the next rise being after the injection of $\frac{1}{30}$ milligramme, when it rose from normal to $1006^{\circ}$. The remaining injections caused apparently no rise of tempera. ture. After the injection of milligramme redness and tenderness were observed at the seat of injection. The sinus healed and the patient was then discharged.

Harley-street, W.

\section{A CASE OF ULCERATIVE ENDOCARDITIS SUCCESSFULLY TREATED WITH ANTI- STREPTOCOCCIC SERUM.}

BY J. W. WASHBOURN, M.D., F.R.C.P. LòND.,

PRISICLAY TO THE LONDON FEVIR HOSPITAL; ASSISTANT PAYSICIAN TO GUY'S HOSPITAL, ETC.

A FEMALE, aged twenty years, was admitted into Guy's Hospital under $\mathrm{my}$ care on Jan. 26th, 1897, complaining of pains in the joints and general malaise. Her father was stated to have died from phthisis and heart disease; otherwise the family history was good. Her occupation was that of a ward maid. She had hitherto enjoyed good health, never having been laid up. The present illness began four days previously with pains in the joints, headache, and shivering. On admission she was found to be a wellnourished, healthy looking young woman. She complained of headache and of pains in the knee and thigh. The heart, lungs, and urine were quite normal and there was no swelling or other evidence of disease in any of the joints. The temperature was $100 \cdot 6^{\circ} \mathrm{F}$, the respirations were 24, and the pulse was 104. On Jan. 27th the temperature, which was taken every four hours throughout the illness, became normal, and remained so for three days. I hoped that she was convalescent, having considered the attack to be one of infinenza, but on Jan. $30 \mathrm{th}$ the temperature again rose, and from this date until March 16th she suffered from irregular pyrexia. With the exception of three days the temperature rose for a few hours every day to $102^{\circ}, 103^{\circ}$, or even $105^{\circ}$, and there were frequent rigors and perspirations. During the whole of this period she was carefully examined every day. The heart, lungs, urine, ears, eyes, vagina, and rectum appeared to be perfectly normal. The pulse varied with the temperature, when the latter was normal the pulse-rate was 80 or 90 per minute. The blood shcwed slight leucocy tosis and slight decrease in bæmoglobin. Some carious teeth were removed, but there was no sign of inflammation in the alveoli. She suffered a good deal from constipation, requiring the frequent use of aperients. In spite of treatment with quinine and salicylate of soda she gradually became worse; the rigors were more frequent, she lost her appetite, and became pale and thin. On March 16th a distinct diastolic bruit developed on the left side of the sternum, its maximum point of intensity being the third intercostal space. It was then quite clear that the pulmonary valve was affected and that the patient was suffering from ulcerative endocarditis. On March 24 th anti-streptococcic serum was first injected, and, with the exception of three days, the injections were continued dai'y until May 25th. The serum employed was that of the British Institute of Preventive Medicine, and it was given in doses of 20 c.c until the last two weeks of treatment, when the dose was gradually diminished. The treatment lasted altogether nine weeks, during which time 1030 c.c. serum were injected in fifty-nine doses. The effect of the treatment is best shown by the chart, which is, unfortunately, too long for publication. During the first fortnight of treatment there were only two slight rigors, but the pyrexia persisted. On April 6tb, a fortnight after the commencement of the treatment, the temperature became normal and remained so until the 26th. By this time the patient was so well that the injections were ordered to be given every other day instead of daily. The effect of this alteration in treatment was that the temperature rose on the $28 \mathrm{th}$ to $99.6^{\circ}$, on the 30 th to $100 \cdot 4^{\circ}$, and on May $3 \mathrm{rd}$ to $100 \cdot 6^{\circ}$. The injections were again given daily, with the result that the temperature fell to normal on May 6th, and continued so urit. July 19th, when the patient was discharged from the hosplal. On May 13th the dose of serum had been reduced to $10 \mathrm{cc}$., and on the 22 nd to 5 c.c. On the 25 h the injections were omitted. The general condition of the patient began to improve five or six days after the commencement of the ivjections, and three weeks later she was to all appearances quite well. On leaving the hospital on July 19th the diastolic bruit in the pulmonary region was still present. Neither now nor on any previous occasion did the heart show any sign of enlargement. The only inconveniences noticed from the frequent injections of the serum were slight pain at the site of injection and an urticarial rash, which appeared on April 1st and lasted a few days. After leaving the hospital the patient went to a convalescent home. She was seen a month later, and appeared to be in perfect health, but the bruit still persisted.

Remarks.-Cases of ulcerative endocarditis treated with serum have been recorded in THE: LANCET by Dr. Harrington Sainsbury, ${ }^{1}$ Dr. A. E. W. Fox, ${ }^{2}$ and Dr. Margaret Pearse. ${ }^{3}$ The above case appears to be more definite in its nature and in the result of the treatment than any of these cases. The patient came under observation a few days after the commencement of the illness. During the first seven weeks she suffered from an ill-defined septicremia without any local manifestation of disease, and there was at no time distinct. evidence of the site of entrance of the contagion. Up to the time when the serum was first injected she was progressively losing ground; and a few days before the commencement of the injection, the palmonary valve became affected. The result of the treatment was most striking and I am quite satisfied that it saved the patient's life. The pyrexia ceased after the first fortnight of treatment, and the general condition very quickly improved. At the end of five weeks the patient seemed so well that the injections were given every other day instead of daily. As a result of this alteration the temperature began to rise, but again subsided when the injections were given daily. Daring the last fortnight of the treatment the quantity of serum injected daily was gradually diminished, and after the final omission of the injections no further rise of temperature occurred. The patient with the exception of pulmonary regurgitation, was perfectly well on leaving the hospital and was seeking a situation a month later. The large amount of serum used is wortby of record. The treatment lasted nine weeks, daring which time 1030 c.c. serum were injected in fifty-nine doses. The only ill-effect was a transient rash. Throughout the case the cardiac symptoms remained in the background, the infective process being the most prominent feature. Such a case as this is the most favourable for serum treatment. In so many cases of nlcerative endocarditis the infective process is engrafted upon old cardiac trouble, and even if the serum controls the septicæmia, the patient has still to contend against cardiac inefficiency. The antistreptococcic serum was used because it was thought that the case was most probably one of streptococcic infection, although we possessed no bacteriological zevidence of the presence of streptococci in the blood.

Cavendish-place, $\mathbf{w}$. 